CRYSTALLOGRAPHIC COMMUNICATIONS

ISSN 2056-9890

Received 31 January 2019

Accepted 5 March 2019

Edited by J. Jasinsk, Keene State College, USA

Keywords: crystal structure; thiourea; chloroacetic acid; thiazolidine; hydrogen bonding.

CCDC reference: 1901297

Supporting information: this article has supporting information at journals.iucr.org/e

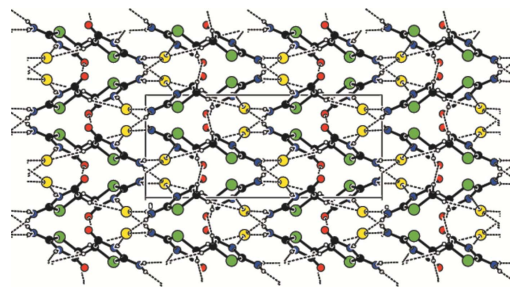

OPEN $\odot$ ACCESS

\section{Redetermination of the crystal structure of 2-oxo-1,3-thiazolidin-4-iminium chloride}

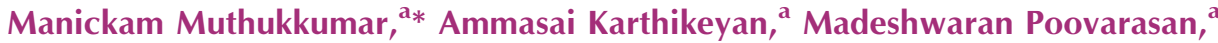 \\ Vadivel Ruckmani, ${ }^{a}$ Dhanakotti Rajaram, ${ }^{b}$ Samson Jegan Jenniferc and Ibrahim \\ Abdul Razakc
}

\begin{abstract}
${ }^{\mathbf{a}}$ Department of Chemistry, Selvamm Arts and Science College, Namakkal, Tamilnadu, India, ${ }^{\mathbf{b}}$ Department of Chemistry,

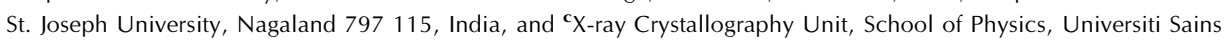
Malaysia, 11800, USM, Penang, Malaysia. *Correspondence e-mail: chemmk70@gmail.com
\end{abstract}

In the redetermination of the title compound, $\mathrm{C}_{3} \mathrm{H}_{5} \mathrm{~N}_{2} \mathrm{OS}^{+} \cdot \mathrm{CI}^{-}$, the asymmetric unit consists of one independent 2-oxo-1,3-thiazolidin-4-iminium cation and one independent chloride anion. The cation interacts with a chloride anion via $\mathrm{N}-$ $\mathrm{H} \cdots \mathrm{Cl}$ hydrogen bonds forming a supramolecular chain along [010]. These supramolecular chains are further extended by weak $\mathrm{C}-\mathrm{H} \cdots \mathrm{Cl}$ and $\mathrm{C}-\mathrm{H} \cdots \mathrm{O}$ interactions, forming a two-dimensional network parallel to (001). The crystal structure is further stabilized by weak $\mathrm{C}-\mathrm{O} \cdots \pi$ interactions, supporting a three-dimensional architecture. The structure was previously determined by Ananthamurthy \& Murthy [Z. Kristallogr. (1975). 8, 356-367] but has been redetermined with higher precision to allow the hydrogen-bonding patterns and supramolecular interactions to be investigated.

\section{Chemical context}

Thiourea and its derivatives are an important group of organic compounds because of their diverse application in fields such as medicine, agriculture, coordination, and analytical chemistry (Saeed et al., 2010, 2014). The complexes with thiourea derivatives expressing biological activity have been successfully screened for various biological actions such as antibacterial, antifungal, anticancer, antioxidant, antiinflammatory, antimalarial, antiviral activity, as anti-HIV agents and also as catalysts (Saeed et al., 2010). Thiazolidine derivatives show antitumor activity as well as a broad range of biological activities including antibactericidal, fungicidal, antiangiogenesis, antidiabetic and antimicrobial (Singh et al., 1981; Saeed \& Florke, 2006; Rizos et al., 2016). Thiourea derivatives are used as phase-change materials for thermal energy storage (Alkan et al., 2011). In addition, metal complexes of thiourea derivatives are also studied for their relationship to NLO materials (Rajasekaran et al., 2003; Ushasree et al., 2000). Thiourea derivatives find applications related to their uses as synthons in supramolecular chemistry (Saeed \& Florke, 2006). Organic and inorganic complexes of thiourea derivatives form well-defined non-covalent supramolecular architectures via multiple hydrogen bonds involving the $\mathrm{N}, \mathrm{S}$ and $\mathrm{O}$ atoms. We report herein the molecular structure and supramolecular architecture of the title salt, $\mathrm{C}_{3} \mathrm{H}_{5} \mathrm{~N}_{2} \mathrm{SO}^{+} \mathrm{CI}^{-}$, (I), formed from the reaction of thiourea with monochloro acetic acid. A determination of this crystal structure was performed by Ananthamurthy \& Murthy (1975). However, while the authors could identify the space group as $P b c a$ and determine the cell 
parameters $[a=9.53(1), b=17.61(5), c=7.71(1) \AA]$, these were not accurate enough to examine the hydrogen-bonding patterns and supramolecular interactions that are described here.

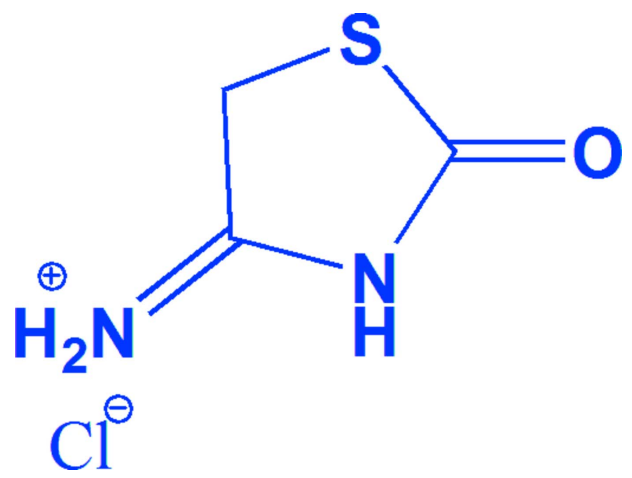

\section{Structural commentary}

The asymmetric unit of the title compound (I) consists of one 2-imino-4-oxo-1,3-thiazolidine cation and one hydrochloride anion (Fig. 1). In the cation, the $\mathrm{C} 3=\mathrm{N} 1$ bond has doublebond character. The $\mathrm{C} 3-\mathrm{N} 1$ and $\mathrm{C} 3-\mathrm{N} 2$ bond distances indicate tautomerism between the amino $\mathrm{N} 1$ and imino $\mathrm{N} 2$ groups. The exocylic bond [C3-N1 = 1.2930 (17) $\AA$ ] is short and its length is comparable with that of the endocylic $\mathrm{C} 3-\mathrm{N} 2$ bond [1.3432 (16) $\AA$ ] , confirming the $\mathrm{C} 3=\mathrm{N} 1$ double-bond assignment. The bond lengths and angles agree with those reported for similar structures (Ananthamurthy \& Murthy, 1975; Xuan et al., 2003; Vedavathi \& Vijayan, 1981).

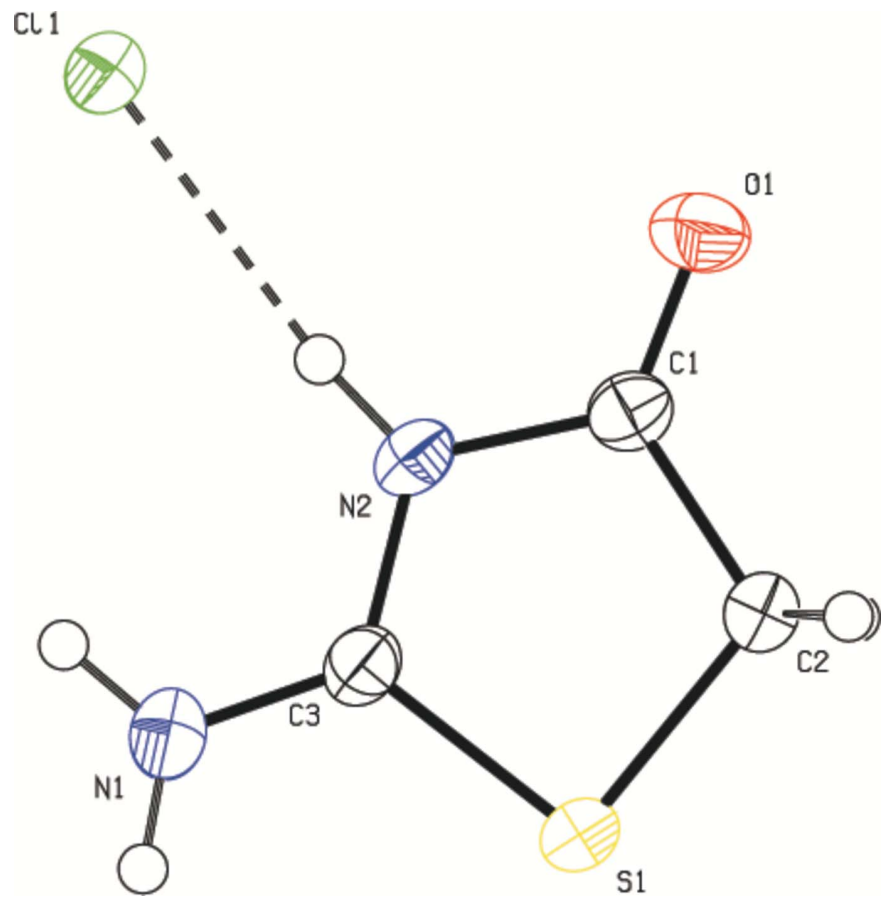

Figure 1

Asymmetric unit of the title compound, showing the atom-numbering scheme and $50 \%$ probability displacements ellipsoids. The dashed line represents the $\mathrm{N} 2-\mathrm{H} 4 \cdots \mathrm{Cl} 1$ hydrogen bond.
Table 1

Hydrogen-bond geometry $\left(\AA,^{\circ}\right)$.

$C g 1$ is the centroid of the $\mathrm{S} 1 / \mathrm{N} 1 / \mathrm{C} 1-\mathrm{C} 3$ ring.

\begin{tabular}{|c|c|c|c|c|}
\hline$D-\mathrm{H} \cdots A$ & $D-\mathrm{H}$ & $\mathrm{H} \cdots A$ & $D \cdots A$ & $D-\mathrm{H} \cdots A$ \\
\hline $\mathrm{N} 1-\mathrm{H} 1 \cdots \mathrm{Cl} 1^{\mathrm{i}}$ & 0.86 & 2.32 & $3.1484(15)$ & 162 \\
\hline $\mathrm{N} 1-\mathrm{H} 3 \cdots \mathrm{Cl}^{\mathrm{ii}}$ & 0.86 & 2.34 & $3.1903(15)$ & 170 \\
\hline $\mathrm{N} 2-\mathrm{H} 4 \cdots \mathrm{Cl} 1$ & 0.86 & 2.26 & $3.1026(12)$ & 166 \\
\hline $\mathrm{C} 2-\mathrm{H} 2 \cdots \mathrm{Cl} 1^{\mathrm{iii}}$ & 0.97 & 2.78 & 3.7137 (14) & 163 \\
\hline $\mathrm{C} 2-\mathrm{H} 5 \cdots \mathrm{O} 1^{\mathrm{iv}}$ & 0.97 & 2.57 & $3.5190(18)$ & 165 \\
\hline $\mathrm{C} 1-\mathrm{O} 1 \cdots C g 1^{\mathrm{v}}$ & $1.20(1)$ & $3.13(1)$ & $3.9430(15)$ & $125(1)$ \\
\hline
\end{tabular}

\section{Supramolecular features}

The 2-imino-4-oxo-1,3-thiazolidine cation interacts with the chlorine anion in the asymmetric unit via the $\mathrm{N} 2-\mathrm{H} 4 \cdots \mathrm{Cl}$ hydrogen bond (Table 1) and with symmetry-related $\mathrm{Cl}^{-}$anions via $\mathrm{N} 1-\mathrm{H} 1 \cdots \mathrm{Cl}$ and $\mathrm{N} 1-\mathrm{H} 3 \cdots \mathrm{Cl}$ hydrogen bonds, forming supramolecular chains along [010] (Fig. 2). The chlorine anion interacts with the $\mathrm{N} 2$ atom and the exocyclic N1 atom of the thiazolidine moiety through the $\mathrm{N} 2-\mathrm{H} 4 \cdots \mathrm{Cl}$

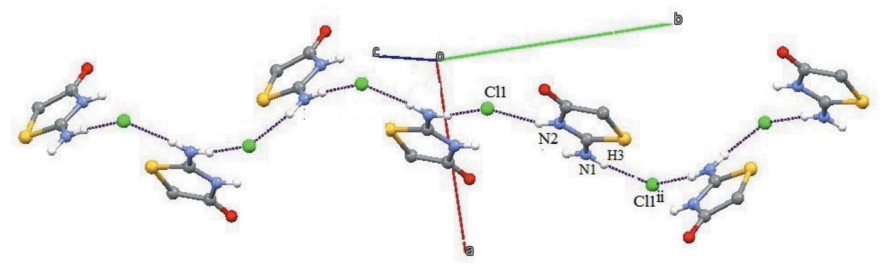

Figure 2

A view of a chain formed by $\mathrm{N}-\mathrm{H} \cdots \mathrm{Cl}$ hydrogen bonds (dashed lines). Symmetry code as in Table 1 .

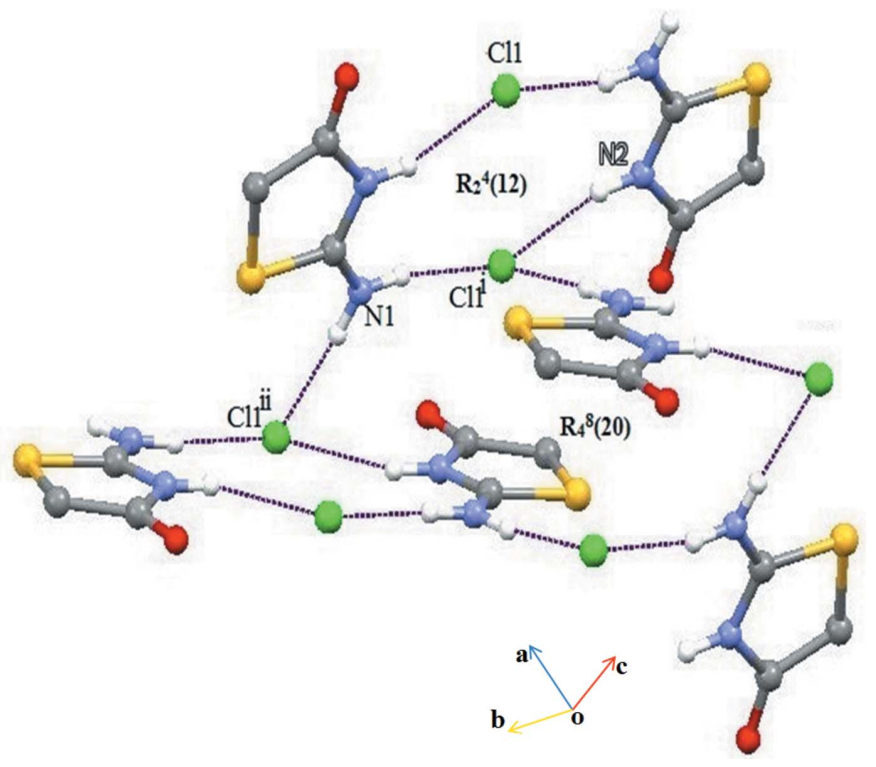

Figure 3

A view of the two supramolecular $R_{2}^{4}(12)$ and $R_{4}^{8}(20)$ ring motifs in the structure of (I), formed by $\mathrm{N}-\mathrm{H} \cdots \mathrm{Cl}$ hydrogen bonds (dashed lines). Symmetry codes are given in Table 1 . 


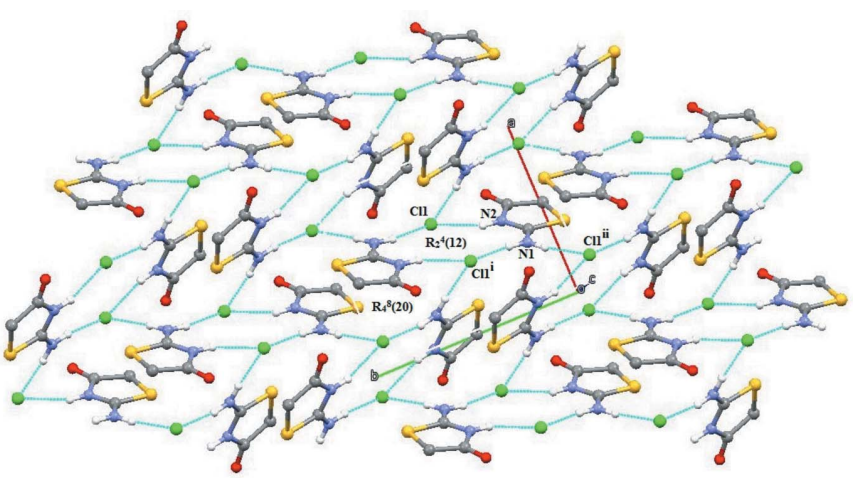

Figure 4

A view of the supramolecular sheet-like structures within the crystal packing of (I). Green dashed lines indicate $\mathrm{N}-\mathrm{H} \cdots \mathrm{Cl}$ hydrogen bonds. Symmetry codes are given in Table 1 .

hydrogen bond and the pair of $\mathrm{N} 1-\mathrm{H} 1 \cdots \mathrm{Cl}$ and $\mathrm{N} 1-$ $\mathrm{H} 3 \cdots \mathrm{Cl}$ hydrogen bonds, forming $R_{2}^{4}(12)$ ring motifs in the [010] plane (Fig. 3). This motif is further connected on the other side by $R_{4}^{8}(20)$ ring motifs, generating a sheet-like structure parallel to (001) (Fig. 4). The supramolecular sheets and crystal packing are further stabilized by weak $\mathrm{C}-\mathrm{H} \cdots \mathrm{Cl}$, $\mathrm{C}-\mathrm{H} \cdots \mathrm{O}$ and $\mathrm{C}=\mathrm{O} \cdots \pi$ interactions (Table 1, Fig. 5). All of these interactions combine to generate a three-dimensional supramolecular architecture (Fig. 6).

\section{Database survey}

The crystal structures of a number of related and substituted thiourea derivatives and thiazoline salts and their metal complexes have also been investigated in a variety of crystalline environments. These include DL-2-amino-2-thiazoline4-carboxylic acid trihydrate (Xuan et al., 2003), 2-amino-1,3thiazoline hydrochloride (Vedavathi \& Vijayan, 1981), $\mathrm{N}$-(4chlorobenzoyl)- $N, N$-diphenylthiourea (Arslan et al., 2003a), 1(4-chloro-benzoyl)-3-naphthalen-1-yl-thiourea (Arslan et al.,

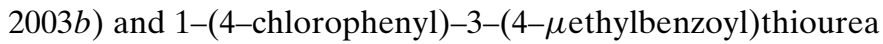

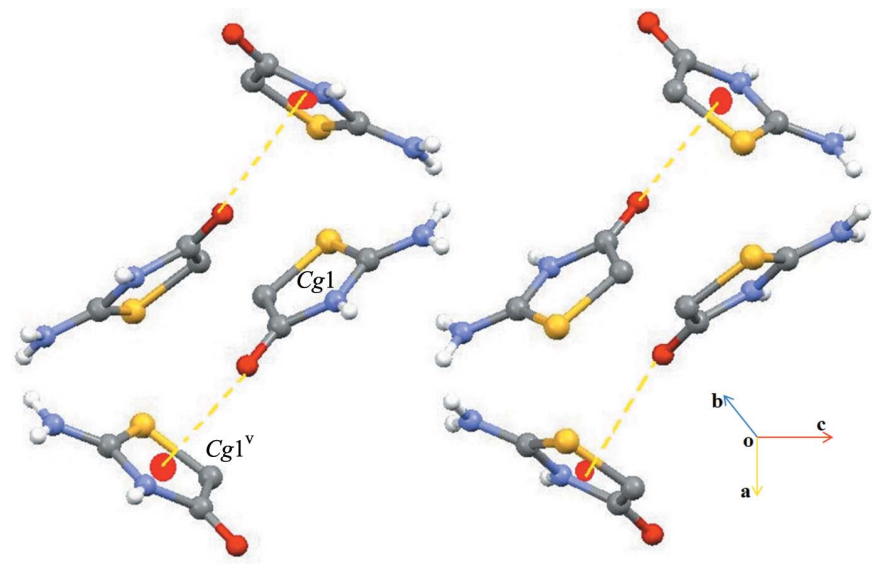

Figure 5

A view of the weak $\mathrm{C}-\mathrm{O} \cdots \pi$ interactions (dashed lines) in (I). $C g 1$ is the centroid of the thiazolidine ring. Symmetry codes are given in Table 1.

Table 2

Experimental details.

Crystal data

Chemical formula

$M_{\mathrm{r}}$

Crystal system, space group

Temperature (K)

$a, b, c(\AA)$

$V\left(\AA^{3}\right)$

$Z$

Radiation type

$\mu\left(\mathrm{mm}^{-1}\right)$

Crystal size (mm)

Data collection

Diffractometer

Absorption correction

$T_{\min }, T_{\max }$

No. of measured, independent and observed $[I>2 \sigma(I)]$ reflections

$R_{\text {int }}$

$(\sin \theta / \lambda)_{\max }\left(\AA^{-1}\right)$

Refinement

$R\left[F^{2}>2 \sigma\left(F^{2}\right)\right], w R\left(F^{2}\right), S$

No. of reflections

No. of parameters

$\mathrm{H}$-atom treatment

$\Delta \rho_{\max }, \Delta \rho_{\min }\left(\mathrm{e} \AA^{-3}\right)$

Computer programs: APEX2 and SAINT (Bruker, 2009), SHELXTL (Sheldrick, 2008), PLATON (Spek, 2009), Mercury (Macrae et al., 2008) and PLATON (Spek, 2009).

(Saeed \& Floörke, 2006). N-H - C Cl hydrogen bonds play a major role in building up the supramolecular architectures of many related crystal structures (for examples, see: Diallo et al., 2014; Yamuna et al., 2014; Plater \& Harrison, 2016; Khongsuk et al., 2015).

\section{Synthesis and crystallization}

Hot ethanol solutions of thiourea $(32 \mathrm{mg})$ and chloro acetic acid $(37 \mathrm{mg})$ were mixed in a 1:1 molar ratio. The resulting solution was warmed over a water bath for half an hour and then kept at room temperature for crystallization. After a week, light-yellow prismatic crystals suitable for single-crystal $\mathrm{X}$-ray analysis were obtained.

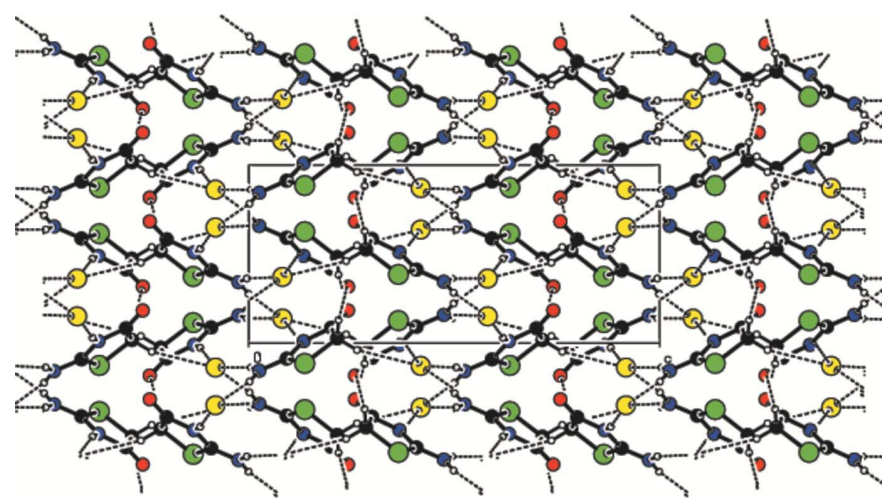

Figure 6

A view of the three-dimensional architecture of the title compound. 


\section{Refinement}

Crystal data, data collection and structure refinement details are summarized in Table 2. All $\mathrm{H}$ atoms were initially located in difference-Fourier maps and were subsequently treated as riding atoms in geometrically idealized positions, with $\mathrm{C}-\mathrm{H}=$ 0.93 and $\mathrm{N}-\mathrm{H}=0.86$ and with $U_{\text {iso }}(\mathrm{H})=1.2 U_{\text {eq }}(\mathrm{C}, \mathrm{N})$.

\section{References}

Alkan, C., Tek, Y. \& Kahraman, D. (2011). Turk. J. Chem. 35, 769777.

Ananthamurthy, R. V. \& Murthy, B. V. R. (1975). Z. Kristallogr. 8, 356-367.

Arslan, H., Flörke, U. \& Külcü, N. (2003b). J. Chem. Crystallogr. 33, 919-924.

Arslan, H., Florke, U. \& Kulucu, N. (2003a). Acta Cryst. E59, o641o642.

Bruker (2009). APEX2, SAINT and SADABS. Bruker AXS Inc., Madison, Wisconsin, USA.

Diallo, W., Diop, L., Plasseraud, L. \& Cattey, H. (2014). Acta Cryst. E70, o618-o619.

Khongsuk, P., Prabpai, S. \& Kongsaeree, P. (2015). Acta Cryst. E71, o608-0609.
Macrae, C. F., Bruno, I. J., Chisholm, J. A., Edgington, P. R., McCabe, P., Pidcock, E., Rodriguez-Monge, L., Taylor, R., van de Streek, J. \& Wood, P. A. (2008). J. Appl. Cryst. 41, 466-470.

Plater, M. J. \& Harrison, W. T. A. (2016). Acta Cryst. E72, 604-607.

Rajasekaran, R., Kumar, R. M., Jayavel, R. \& Ramasamy, P. (2003). J. Cryst. Growth, 252, 317-327.

Rizos, C. V., Kei, A. \& Elisaf, M. S. (2016). Arch. Toxicol. 90, 18611881.

Saeed, A. \& Flörke, U. (2006). Acta Cryst. E62, o2403-o2405.

Saeed, A., Flörke, U. \& Erben, M. F. (2014). J. Sulfur Chem. 35, 318355.

Saeed, S., Rashid, N., Jones, P. G., Ali, M. \& Hussain, R. (2010). Eur. J. Med. Chem. 45, 1323-1331.

Sheldrick, G. M. (2008). Acta Cryst. A64, 112-122.

Singh, S. P., Parmar, S. S., Raman, K. \& Stenberg, V. I. (1981). Chem. Rev. 81, 175-203.

Spek, A. L. (2009). Acta Cryst. D65, 148-155.

Ushasree, P. M., Muralidharan, R., Jayavel, R. \& Ramasamy, P. J. (2000). J. Cryst. Growth, 218, 365-371.

Vedavathi, B. M. \& Vijayan, K. (1981). Acta Cryst. B37, 475-477.

Xuan, R.-C., Hu, W.-X., Yang, Z.-Y. \& Xuan, R.-R. (2003). Acta Cryst. E59, o1707-o1709.

Yamuna, T. S., Jasinski, J. P., Kaur, M., Anderson, B. J. \& Yathirajan, H. S. (2014). Acta Cryst. E70, 203-206. 


\section{supporting information}

Acta Cryst. (2019). E75, 443-446 [https://doi.org/10.1107/S2056989019003189]

\section{Redetermination of the crystal structure of 2-oxo-1,3-thiazolidin-4-iminium chloride}

\section{Manickam Muthukkumar, Ammasai Karthikeyan, Madeshwaran Poovarasan, Vadivel Ruckmani, Dhanakotti Rajaram, Samson Jegan Jennifer and Ibrahim Abdul Razak}

\section{Computing details}

Data collection: APEX2 (Bruker, 2009); cell refinement: SAINT (Bruker, 2009); data reduction: SAINT (Bruker, 2009); program(s) used to solve structure: SHELXTL (Sheldrick, 2008); program(s) used to refine structure: SHELXTL (Sheldrick, 2008); molecular graphics: PLATON (Spek, 2009) and Mercury (Macrae et al., 2008); software used to prepare material for publication: PLATON (Spek, 2009).

2-Oxo-1,3-thiazolidin-4-iminium chloride

Crystal data

$\mathrm{C}_{3} \mathrm{H}_{5} \mathrm{~N}_{2} \mathrm{OS}^{+} \cdot \mathrm{Cl}^{-}$

$M_{r}=152.60$

Orthorhombic, $\mathrm{Pbca}$

$a=7.5106(11) \AA$

$b=9.3140(13) \AA$

$c=17.343(3) \AA$

$V=1213.2(3) \AA^{3}$

$Z=8$

$F(000)=624$

Data collection

Bruker SMART APEXII DUO CCD area detector diffractometer

Radiation source: fine-focus sealed tube Graphite monochromator phi and $\omega$ scans

Absorption correction: multi-scan

(SADABS; Bruker, 2009)

$T_{\min }=0.683, T_{\max }=0.832$

\section{Refinement}

Refinement on $F^{2}$

Least-squares matrix: full

$R\left[F^{2}>2 \sigma\left(F^{2}\right)\right]=0.027$

$w R\left(F^{2}\right)=0.077$

$S=1.05$

1798 reflections

73 parameters

0 restraints
$D_{\mathrm{x}}=1.671 \mathrm{Mg} \mathrm{m}^{-3}$

Mo $K \alpha$ radiation, $\lambda=0.71073 \AA$

Cell parameters from 1800 reflections

$\theta=2.4-30.2^{\circ}$

$\mu=0.87 \mathrm{~mm}^{-1}$

$T=296 \mathrm{~K}$

Prism, yellow

$0.54 \times 0.45 \times 0.25 \mathrm{~mm}$

7517 measured reflections 1798 independent reflections 1554 reflections with $I>2 \sigma(I)$

$R_{\text {int }}=0.020$

$\theta_{\text {max }}=30.2^{\circ}, \theta_{\text {min }}=2.4^{\circ}$

$h=-10 \rightarrow 10$

$k=-10 \rightarrow 13$

$l=-24 \rightarrow 20$

Primary atom site location: structure-invariant direct methods

Secondary atom site location: difference Fourier map

Hydrogen site location: inferred from neighbouring sites

$\mathrm{H}$-atom parameters constrained 
$w=1 /\left[\sigma^{2}\left(F_{\mathrm{o}}^{2}\right)+(0.0384 P)^{2}+0.4007 P\right]$

where $P=\left(F_{\mathrm{o}}^{2}+2 F_{\mathrm{c}}^{2}\right) / 3$

$(\Delta / \sigma)_{\max }=0.001$

$$
\Delta \rho_{\max }=0.33 \text { e } \AA^{-3}
$$

\section{Special details}

Geometry. All esds (except the esd in the dihedral angle between two 1.s. planes) are estimated using the full covariance matrix. The cell esds are taken into account individually in the estimation of esds in distances, angles and torsion angles; correlations between esds in cell parameters are only used when they are defined by crystal symmetry. An approximate (isotropic) treatment of cell esds is used for estimating esds involving l.s. planes.

Refinement. Refinement of $\mathrm{F}^{2}$ against ALL reflections. The weighted R-factor $\mathrm{wR}$ and goodness of fit $\mathrm{S}$ are based on $\mathrm{F}^{2}$, conventional R-factors $R$ are based on $F$, with $F$ set to zero for negative $\mathrm{F}^{2}$. The threshold expression of $\mathrm{F}^{2}>2 \operatorname{sigma}\left(\mathrm{F}^{2}\right)$ is used only for calculating R-factors(gt) etc. and is not relevant to the choice of reflections for refinement. R-factors based on $\mathrm{F}^{2}$ are statistically about twice as large as those based on F, and R- factors based on ALL data will be even larger.

Fractional atomic coordinates and isotropic or equivalent isotropic displacement parameters $\left(\hat{A}^{2}\right)$

\begin{tabular}{lllll}
\hline & $x$ & $y$ & $z$ & $U_{\text {iso }} / U_{\text {eq }}$ \\
\hline S1 & $0.37439(5)$ & $-0.01239(4)$ & $0.86421(2)$ & $0.03155(11)$ \\
O1 & $0.67766(16)$ & $0.25652(11)$ & $0.75866(7)$ & $0.0389(2)$ \\
N1 & $0.3380(2)$ & $0.18329(15)$ & $0.97467(7)$ & $0.0409(3)$ \\
H1 & 0.3567 & 0.2663 & 0.9949 & $0.049^{*}$ \\
H3 & 0.2747 & 0.1209 & 0.9988 & $0.049^{*}$ \\
C3 & $0.40497(18)$ & $0.15240(14)$ & $0.90803(7)$ & $0.0270(3)$ \\
C2 & $0.51618(18)$ & $0.03829(13)$ & $0.78499(7)$ & $0.0272(3)$ \\
H5 & 0.6172 & -0.0263 & 0.7817 & $0.033^{*}$ \\
H2 & 0.4507 & 0.0336 & 0.7368 & $0.033^{*}$ \\
C1 & $0.57886(18)$ & $0.18932(13)$ & $0.79961(7)$ & $0.0264(3)$ \\
N2 & $0.50511(14)$ & $0.24392(11)$ & $0.86677(6)$ & $0.0268(2)$ \\
H4 & 0.5224 & 0.3312 & 0.8812 & $0.032^{*}$ \\
C11 & $0.63837(5)$ & $0.54422(4)$ & $0.91839(2)$ & $0.03440(11)$ \\
\end{tabular}

Atomic displacement parameters $\left(\AA^{2}\right)$

\begin{tabular}{lllllll}
\hline & $U^{11}$ & $U^{22}$ & $U^{33}$ & $U^{12}$ & $U^{13}$ & $U^{23}$ \\
\hline S1 & $0.0393(2)$ & $0.02303(17)$ & $0.03232(19)$ & $-0.00807(13)$ & $0.00667(13)$ & $-0.00457(12)$ \\
O1 & $0.0438(6)$ & $0.0310(5)$ & $0.0418(6)$ & $-0.0042(5)$ & $0.0099(5)$ & $0.0066(4)$ \\
N1 & $0.0593(9)$ & $0.0310(6)$ & $0.0325(6)$ & $-0.0057(6)$ & $0.0111(6)$ & $-0.0077(5)$ \\
C3 & $0.0311(6)$ & $0.0224(5)$ & $0.0275(6)$ & $-0.0007(5)$ & $-0.0013(5)$ & $-0.0025(4)$ \\
C2 & $0.0301(6)$ & $0.0255(6)$ & $0.0261(6)$ & $-0.0020(5)$ & $0.0015(5)$ & $-0.0030(4)$ \\
C1 & $0.0281(6)$ & $0.0223(5)$ & $0.0289(6)$ & $0.0021(5)$ & $-0.0022(5)$ & $0.0028(4)$ \\
N2 & $0.0312(5)$ & $0.0188(5)$ & $0.0304(5)$ & $-0.0014(4)$ & $-0.0018(4)$ & $-0.0022(4)$ \\
C11 & $0.0453(2)$ & $0.02841(18)$ & $0.02950(18)$ & $-0.00821(13)$ & $0.00436(13)$ & $-0.00460(12)$ \\
\hline
\end{tabular}

Geometric parameters $\left(\stackrel{A}{\circ}{ }^{\circ}\right)$

\begin{tabular}{llll}
\hline $\mathrm{S} 1-\mathrm{C} 3$ & $1.7280(13)$ & $\mathrm{C} 3-\mathrm{N} 2$ & $1.3432(16)$ \\
$\mathrm{S} 1-\mathrm{C} 2$ & $1.8013(14)$ & $\mathrm{C} 2-\mathrm{C} 1$ & $1.5049(18)$ \\
$\mathrm{O} 1-\mathrm{C} 1$ & $1.2027(17)$ & $\mathrm{C} 2-\mathrm{H} 5$ & 0.9700 \\
$\mathrm{~N} 1-\mathrm{C} 3$ & $1.2930(17)$ & $\mathrm{C} 2-\mathrm{H} 2$ & 0.9700
\end{tabular}




$\begin{array}{llll}\mathrm{N} 1-\mathrm{H} 1 & 0.8600 & \mathrm{C} 1-\mathrm{N} 2 & 1.3865(17) \\ \mathrm{N} 1-\mathrm{H} 3 & 0.8600 & \mathrm{~N} 2-\mathrm{H} 4 & 0.8600 \\ \mathrm{C} 3-\mathrm{S} 1-\mathrm{C} 2 & 91.38(6) & \mathrm{C} 1-\mathrm{C} 2-\mathrm{H} 2 & 110.2 \\ \mathrm{C} 3-\mathrm{N} 1-\mathrm{H} 1 & 120.0 & \mathrm{~S} 1-\mathrm{C} 2-\mathrm{H} 2 & 110.2 \\ \mathrm{C} 3-\mathrm{N} 1-\mathrm{H} 3 & 120.0 & \mathrm{H} 5-\mathrm{C} 2-\mathrm{H} 2 & 108.5 \\ \mathrm{H} 1-\mathrm{N} 1-\mathrm{H} 3 & 120.0 & \mathrm{O} 1-\mathrm{C} 1-\mathrm{N} 2 & 123.48(12) \\ \mathrm{N} 1-\mathrm{C} 3-\mathrm{N} 2 & 123.56(12) & \mathrm{O} 1-\mathrm{C} 1-\mathrm{C} 2 & 125.45(12) \\ \mathrm{N} 1-\mathrm{C} 3-\mathrm{S} 1 & 122.62(11) & \mathrm{C} 3-\mathrm{N} 2-\mathrm{C} 1 & 111.06(11) \\ \mathrm{N} 2-\mathrm{C} 3-\mathrm{S} 1 & 113.82(9) & \mathrm{C} 3-\mathrm{N} 2-\mathrm{H} 4 & 116.00(11) \\ \mathrm{C} 1-\mathrm{C} 2-\mathrm{S} 1 & 107.55(9) & \mathrm{C} 1-\mathrm{N} 2-\mathrm{H} 4 & 122.0 \\ \mathrm{C} 1-\mathrm{C} 2-\mathrm{H} 5 & 110.2 & & 174.68(14) \\ \mathrm{S} 1-\mathrm{C} 2-\mathrm{H} 5 & 110.2 & \mathrm{~N} 1-\mathrm{C} 3-\mathrm{N} 2-\mathrm{C} 1 & -4.87(15) \\ & & \mathrm{S} 1-\mathrm{C} 3-\mathrm{N} 2-\mathrm{C} 1 & -176.60(14) \\ \mathrm{C} 2-\mathrm{S} 1-\mathrm{C} 3-\mathrm{N} 1 & -176.63(14) & \mathrm{O} 1-\mathrm{C} 1-\mathrm{N} 2-\mathrm{C} 3 & 4.40(16) \\ \mathrm{C} 2-\mathrm{S} 1-\mathrm{C} 3-\mathrm{N} 2 & 2.92(11) & \mathrm{C} 2-\mathrm{C} 1-\mathrm{N} 2-\mathrm{C} 3 & \\ \mathrm{C} 3-\mathrm{S} 1-\mathrm{C} 2-\mathrm{C} 1 & -0.44(10) & & \\ \mathrm{S} 1-\mathrm{C} 2-\mathrm{C} 1-\mathrm{O} 1 & 179.02(12) & -2.00(13) & \end{array}$

Hydrogen-bond geometry $\left(\AA,{ }^{\circ}\right)$

$\mathrm{Cg} 1$ is the centroid of the $\mathrm{S} 1 / \mathrm{N} 1 / \mathrm{C} 1-\mathrm{C} 3$ ring.

\begin{tabular}{lllll}
\hline$D-\mathrm{H} \cdots A$ & $D-\mathrm{H}$ & $\mathrm{H} \cdots A$ & $D \cdots A$ & $D-\mathrm{H} \cdots A$ \\
\hline $\mathrm{N} 1-\mathrm{H} 1 \cdots \mathrm{C} 11^{\mathrm{i}}$ & 0.86 & 2.32 & $3.1484(15)$ & 162 \\
$\mathrm{~N} 1-\mathrm{H} 3 \cdots \mathrm{C} 11^{1 i}$ & 0.86 & 2.34 & $3.1903(15)$ & 170 \\
$\mathrm{~N} 2-\mathrm{H} 4 \cdots \mathrm{C} 11$ & 0.86 & 2.26 & $3.1026(12)$ & 166 \\
$\mathrm{C} 2-\mathrm{H} 2 \cdots \mathrm{C} 11^{\mathrm{iii}}$ & 0.97 & 2.78 & $3.7137(14)$ & 163 \\
$\mathrm{C} 2-\mathrm{H} 5 \cdots \mathrm{O} 1^{\mathrm{iv}}$ & 0.97 & 2.57 & $3.5190(18)$ & 165 \\
$\mathrm{C} 1-\mathrm{O} 1 \cdots \mathrm{Cg} 1^{\mathrm{v}}$ & $1.20(1)$ & $3.13(1)$ & $3.9430(15)$ & $125(1)$
\end{tabular}

Symmetry codes: (i) $-x+1,-y+1,-z+2$; (ii) $x-1 / 2,-y+1 / 2,-z+2$; (iii) $-x+1, y-1 / 2,-z+3 / 2$; (iv) $-x+3 / 2, y-1 / 2, z$; (v) $x+1 / 2, y,-z+3 / 2$. 